\section{Rotating Hinge Total Knee Arthroplasty RT-PLUS Solution: A Clinical and Radiographic Follow-} Up

\begin{abstract}
Background: In total knee arthroplasty (TKA), there is still controversy surrounding the preferable prosthetic design and required amount of constraint. Indication for rotating hinge TKA includes primary and revision arthroplasty, with contradictory results. We present the first medium- to long-term results with the rotating hinge knee prosthesis RT-PLUS SOLUTION (Smith \& Nephew Orthopaedics, Baar, Switzerland).

Methods: A retrospective, non-randomized, observational, single centre study was conducted, in which 146 total knee replacements were performed in 136 patients between 2003 and 2012 using the RTPLUS SOLUTION rotating hinge knee (Smith \& Nephew Orthopaedics, Baar, Switzerland). In 77 of the 146 cases, the system was used as a primary implant. The mean age of the population at time of index surgery was $76.3 \pm 10.4$ years.

Results: At final follow-up, 62 patients ( 62 knees) were available for clinical and radiological follow-up after a mean of $6.8 \pm 2.2$ years. There were three revisions due to mechanical complications (loosening) and one septic revision. Ten year cumulative implant survival was $89.4 \%$ (95\% confidence interval, 68.8\%-96.7\%). Radiolucent lines were observed in 36 cases. They were, however, clinically asymptomatic and without therapeutic consequences in all cases. Clinical outcome and patient satisfaction outcomes were in line with previous investigations.

Conclusion: To our knowledge, these are the first medium- to longterm follow-up results with the RT-PLUS Solution. This rotating hinge knee shows good clinical and radiographical results and good longevity.
\end{abstract}

\section{Introduction}

As the number of primary total knee arthroplasties (TKAs) being performed continues to increase, orthopedic surgeons are faced with a growing number of difficult cases with severe deformity due to ligamentous instability and metaphyseal bone loss [1].

Historically, such cases have been managed using hinge knee prostheses with a fixed axis to restore knee function and correct limb alignment [2]. Disappointing long-term results with high rates of aseptic and septic loosening prompted initiatives to further refine the design of these prostheses [3]. The rotating hinge total knee prosthesis was introduced in 1982 [4]. The additional rotation around the tibial axis is thought to reduce the shear stress around the bone-cement interface $[5,6]$.

Several authors have investigated different types of rotating hinge implants, in some cases with controversial conclusions [7-11]. Up until today there is still controversy surrounding the preferable prosthetic design and required amount of constraint $[12,13]$.

In this retrospective analysis we hypothesized that the use of the rotating hinge RT-PLUS SOLUTION knee implant (Smith \& Nephew Orthopaedics AG, Baar, Switzerland) in primary and/or

\section{Journal of}

Orthopedics \& Rheumatology

\section{Kai Wilke, Daniel Kunze, Peggy Hommel and Hagen Hommel*}

Krankenhaus Märkisch Oderland GmbH, BT Wriezen, Klinik für Orthopädie, Sportmedizin und Rehabilitation, Wriezen, Germany

\section{*Address for Correspondence}

Hagen Hommel, MD, Krankenhaus Märkisch Oderland $\mathrm{GmbH}$, Sonnenburger Weg 3, 16269 Wriezen, Germany, Tel: +4933456 - 40401; Fax: +4933456 - 40402; E-mail:H.Hommel@khmol.de

Submission: 14 December 2015

Accepted: 09 February 2016

Published: 15 February 2016

Copyright: () 2016 Wilke K, et al. This is an open access article distributed under the Creative Commons Attribution License, which permits unrestricted use, distribution, and reproduction in any medium, provided the original work is properly cited.

revision total knee arthroplasty would result in good clinical and radiological results. As far as the authors are aware, this is the first report on medium- to long-term results with this implant in the peerreviewed orthopaedic literature.

\section{Materials and Methods}

A retrospective, non-randomized, single centre, medium- to long-term observational study was conducted in an unselected, consecutive series of patients who had received the RT-PLUS SOLUTION rotating hinge knee. All 136 patients (146 prostheses), who had received this rotating hinge implant design between January 2003 and December 2012, were reviewed. Ethics committee approval was obtained prior to study commencement. Patients who refused to pro-vide informed consent were excluded, as were patients who did not receive the device of interest.

The RT-PLUS SOLUTION-type knee implant (Figure 1) is available in two versions: a standard monobloc version with an integrated stem for cemented implantation, and a modular version. In addition, both cemented stems, made from $\mathrm{CoCrMo}$, and cementless Ti6Al4V stems are available. The modular stems are connected to the femoral and tibial components via a cone connection secured with screws. We used cemented stems exclusively. No offset stems were used. The decision to use a standard or modular system was always intraoperatively.

Patients who presented with severe malalignment (varus or valgus angles $>18^{\circ}$ ), severe bone loss, ligamentous instability, and flexion contracture $\left(>10^{\circ}\right)$ were advised to undergo rotating hinge TKA.

In varus cases a medial parapatellar approach was used, while a lateral approach with release of the iliotibial band was employed in valgus patients. All operations were carried out by a single experienced surgeon (H.H.).

In 77 of the 146 cases the system was used as a primary implant; the percentage of primary TKAs that was performed with the current 


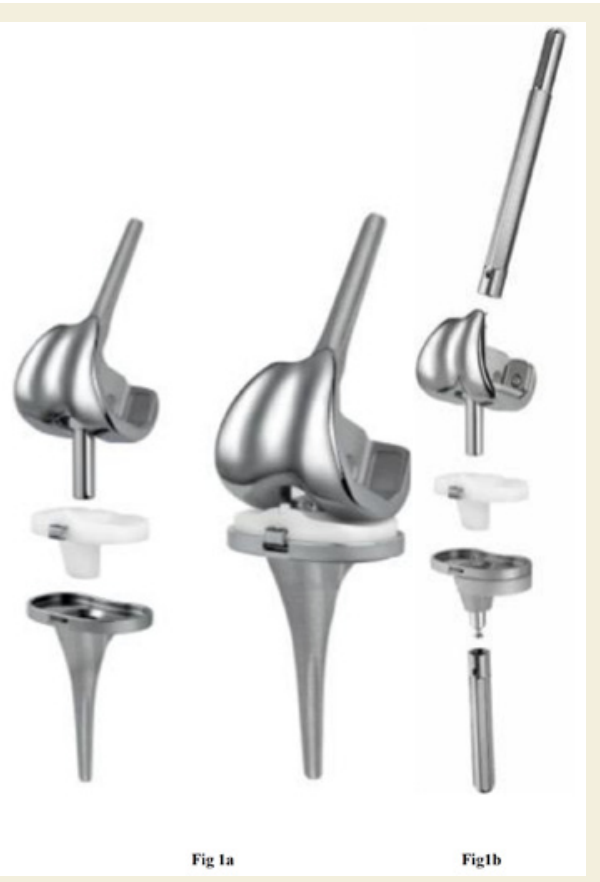

Figures 1a and 1b: Systems RT-PLUS Solution (a) and RT-PLUS Solution Modular (b).

rotating hinge design was approximately $3.5 \%$. In the remaining 69 of the 146 cases the system was used as a revision implant. The average age \pm standard deviation (SD) of the population at the time of operation was $76.3 \pm 10.4$ years, and 88 patients $(64.7 \%)$ were female.

Each patient was contacted by telephone and invited for followup assessment. Minimal implant data was acquired from patients who refused or were unable to attend the follow-up appointment in our clinic, including for those who had ceased during the course of the study. Implant status data was obtained either directly from the patient or from a patient's relative, his/her general practitioner, or home care nursery.

The Knee Society Clinical Score (K-KSS) and Knee Society Function Score (F-KSS) [14], alongside the Hospital for Special Surgery Score (HSS) [15] were recorded for the clinical evaluation. The range of motion of the knee was recorded in all cases with a goniometer. The subjective assessment employed the visual analogue scale for pain (level 1: least pain, level 10: maximum pain) and the general satisfaction assessment of activities of daily living (1: very satisfied, 2: satisfied, 3: moderately satisfied, 4: dissatisfied).

Radiographic follow-up was carried out in all patients who attended follow-up. Anterior-posterior and lateral views were recorded, as well as a full-leg standing x-ray. The radiological assessment was based on the occurrence of radiolucent lines, signs of loosening and implants migration.

Continuous data are presented as the mean and range. Categorical variables are presented as frequencies and percentages. Implant survivorship was calculated using Kaplan- Meier analysis [16], with revision for any reason and revision for aseptic loosening as endpoints of interest. Kaplan-Meier analysis was performed with patients for whom the revision status was known. Only patients who were lost to follow-up were excluded. Sample size estimation was based on a hypothesized revision rate of $15 \%$ at 10 -year follow-up, although we hypothesized that a survival rate of at least $95 \%$ can reasonably be expected. Based on an algorithm defined by Lawless [17], and setting $\alpha$ at 0.05 and $\beta$ at 0.2 , it was estimated that the study would require a population of 96 knees at 10-year follow-up. Under the assumption of approximately $50 \%$ censoring, a baseline study population of $\approx 145$ knees would therefore be required.

STATA 12.1 (StataCorp, College Station, TX, USA) was used for the statistical analysis.

\section{Results}

Radiographic and clinical follow-up was carried out in 62 patients (62 knees), with an average follow-up period of $6.8 \pm 2.2$ years (range, 2.8-11.7 years). In all, 74 patients ( 84 knees) did not attend the followup: 3 patients ( 4 knees) due to a (partial) revision of the implant, 14 patients ( 16 knees) had died by the date of the last follow-up appointment, 42 patients ( $48 \mathrm{knees}$ ) could not be examined due to multimorbidity, 13 patients (14 knees) did not give their consent to participate in the study, and 2 patients ( 2 knees) were recorded as lost to follow-up (Table 1).

The 62 patients who were followed up comprised 15 men and 47 women. The average age at the time of follow-up was $74.1 \pm 9.9$ years, average BMI was $30.2 \pm 5.2 \mathrm{~kg} / \mathrm{m}^{2}$. In 28 of the 62 cases the system had been used as a primary implant: in 18 cases due to a varus deformity, in 6 cases due to a valgus deformity and in 4 cases due to instability. In the other 34 cases the system had been employed as a revision implant: in 5 cases due to an infection, in 16 cases due to loosening, in 1 case due to a periprosthetic fracture, in 7 cases after instability and in 5 cases due to malfunctioning of the primary implant.

In the whole population of 146 prostheses, device removal was required in two primary cases and two revision cases. In two cases an aseptically loosened tibial component had to be replaced, while in another case only the femoral component needed to be revised. One prosthesis had to be removed due to sepsis. Thus, the cumulative survival rate for the system, with revision for any reason as endpoint of interest, was $99.2 \%$ (95\% confidence interval (CI), 94.5\%-99.9\%) after 5 years, and $89.4 \%$ (95\% CI, 68.8\%-96.7\%) after 10 years (Figure 2). For the endpoint of revision due to aseptic loosening, the cumulative survival rate was $99.2 \%$ (95\% CI, 94.5\%-99.9\%) after 5 years, and 90.9\% (95\% CI, 68.7\%-97.6\%) after 10 years.

Of the 62 patients (62 knees) who returned to the hospital for a

Table 1: Follow-up status of patients and implants.

\begin{tabular}{|c|c|c|}
\hline Follow-up status & Patients & Implants \\
\hline a. Number at start of study & 136 & 146 \\
\hline Excluded from follow-up: & & \\
\hline b. Died & 14 & 16 \\
\hline c. (Partial) explantation of the prosthesis & 3 & 4 \\
\hline d. No follow-up, multimorbidity & 42 & 48 \\
\hline e. Study participation declined, without revision & 13 & 14 \\
\hline f. Lost to follow-up, status of prosthesis unknown & 2 & 2 \\
\hline g. Number with clinical and radiological follow-up & 62 & 62 \\
\hline (=a-[b+c+d+e+f]) & & \\
\hline
\end{tabular}


Citation: Wilke K, Peggy D, Hommel P, Hommel H. Rotating Hinge Total Knee Arthroplasty RT-PLUS Solution: A Clinical and Radiographic Follow-Up. J Orthopedics Rheumatol. 2016; 3(1): 4.

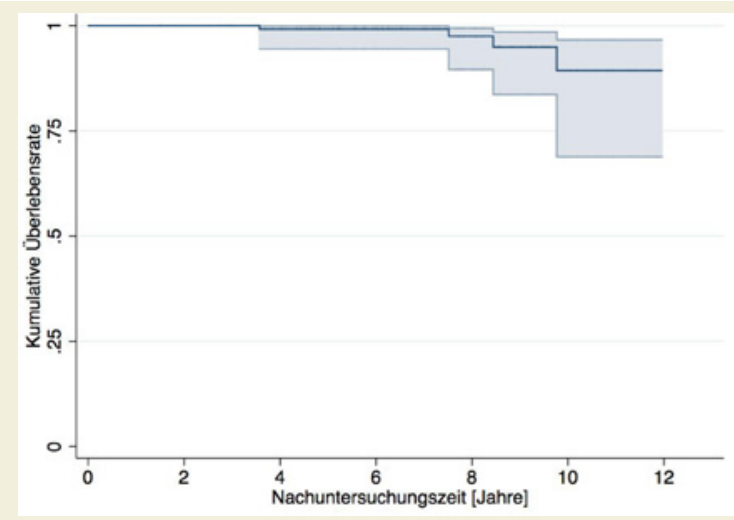

Figure 2: Kaplan-Meier survival plot of revision of any component for any reason.

follow-up assessment of the knee, range of motion was $117.5^{\circ} \pm 16.9^{\circ}$. The K-KSS was $89.2 \pm 12.6$, the F-KSS $75.2 \pm 27.3$, and the HSS was $86.6 \pm 13.1$. With a score of $1.6 \pm 0.8$, all examined patients stated that they were very satisfied or satisfied with the implanted knee prosthesis, with a mean pain score of $1.7 \pm 2.0$ on the visual analogue scale for pain.

No radiological signs of loosening or implant migration were found in any patient (Figure 3), although in 36 knees (58.1\%) asymptomatic radiolucent lines were observed. Of those, 14 (22.6\%) involved the femoral component and 22 (35.5\%) the tibial component. None of the radiolucent lines was continuous, and all the lines measured less than $1.5 \mathrm{~mm}$ in width. They were also all clinically asymptomatic and without any therapeutic consequences at the time of follow-up.

\section{Discussion}

There has been an increasing interest in the use of rotating hinge knee prostheses. With the growing incidence of osteoarthritis of the knee and the increasing incidence of patients presenting with severe deformities or bone defects situations, rotating hinge implants are thought to play an increasingly important role. However, there is paucity and variation in available data regarding both indications for use as clinical outcome for this type of knee implant. On the whole there appears to be no unanimity on the indications for their use. Some authors view them solely as revision prostheses and less as prostheses for primary implantation [18-20], one article referring, by way of explanation, to an increased risk of loosening compared to unhinged implants [18]. Agreement does exist however concerning the indications for major bone defects and gross ligament instability, as well as large varus and valgus deformities $[7,19,21]$. Only a few articles to date reported long term follow-up, and the numbers of cases involved are usually small. As a result, scant meaningful data are currently available from reliable long-term observational studies.

As regards implant failure, one case of septic loosening and three cases of aseptic loosening leading to revision of the implanted prostheses were observed in our study. This corresponds to a 10-year implant survival rate of $89.4 \%$. The literature describes varying failure rates for rotating hinge systems $[8,18,19,22,23]$. Müller et al. used the same knee system for primary procedures and found a cumulative survival rate of $92.9 \%$ after 5 years with revision for any reason as the end point [22]. In a cohort consisting of revision cases, Gudnason et al. observed a comparable 10-year survival rate of $89.2 \%$ due to aseptic loosening. When other revisions of the in-situ prosthesis are included, the survival rate was only $65.1 \%$ [8]. In primary cases, Bistolfi et al. reported a cumulative implant survival rate of $79.8 \%$ and $75.8 \%$ at 10 and 15 years, respectively [18], while for revision cases a survival rate of $80.4 \%$ at 12.5 years was found [19]. Finally, Giurea et al. reported $89.4 \%$ survival at 2 years in a mixed cohort consisting of primary and revision cases [23].

We rated the determined range of motion $\left(117.5^{\circ} \pm 16.9^{\circ}\right)$ as very good. This is in accordance with the literature and is reflected in the high level of patient satisfaction and low pain scores (VAS) [20,24].

The analysis of the clinical scores points to an above-average outcome. Differing results on this subject are reported in the literature. Some authors describe good results. Bistolfi et al. reported a HSS of 88.4 at a mean follow-up of 13 years [20]; Sanguineti et al. found a K-KSS and F-KKS of 94.2 and 78.7, respectively, at a mean follow-up of 3.5 years [25]; Neumann et al. found a K-KSS and F-KKS of 91 and 85, at a mean follow-up of 4.7 years [6]. Gudnason et al. however, reported moderate results only: the HSS was 67, and the K-KSS and F-KKS were 85 and 29, respectively, at a mean follow-up of 8.8 years [8].

With regard to clinical outcome after rotating hinge knee arthroplasty, it must be borne in mind that, in general, functional outcome will be lower compared with outcome after primary TKA, as results of the comparably older age of the population, alongside the comorbidity status of this patient group. In our view this enhances the results of the current study.

There are several limitations to our study. All of the procedures were performed by a single surgeon in a single institution. Consequently, the findings are not readily generalizable. The average follow-up period of this retrospective study was relatively short, and no control group was employed for this study. In addition, the study results are potentially limited by the small sample sizes and

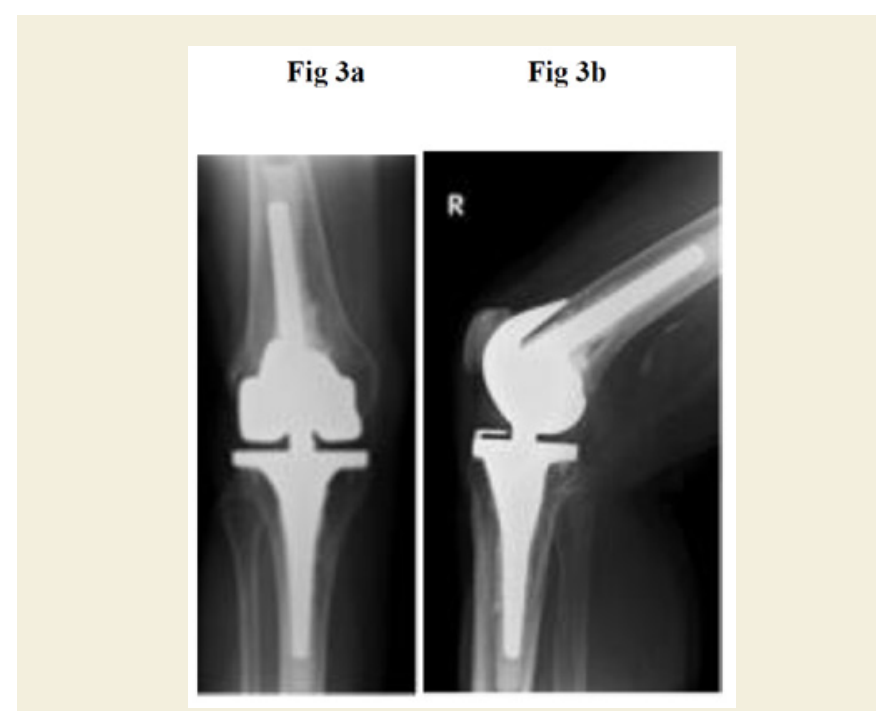

Figures 3a and 3b: 68-year-old female patient, good implant position with no signs of loosening at the 6 -year follow-up. 
Citation: Wilke K, Peggy D, Hommel P, Hommel H. Rotating Hinge Total Knee Arthroplasty RT-PLUS Solution: A Clinical and Radiographic Follow-Up. J Orthopedics Rheumatol. 2016; 3(1): 4.

the heterogeneity in our series in terms of indication for surgery. Moreover, a large proportion of patients were not available for the clinical and radiological follow-up. The attrition rate was higher than anticipated, which is thought to be due to the relatively high age of the study population, alongside the large proportion of patients with significant comorbidities. However, the lost to follow-up rate of two patients was not unduly large. We did not evaluate patient reported outcome measures such as WOMAC (Western Ontario and McMaster Universities Osteoarthritis Index); this is obviously another important aspect to consider for future research since restoration of physical activity is an important surgical objective.

If knee stability cannot be achieved with normal surface replacement, the use of rotating hinge knee prosthesis is justified [26-28]. We agree with Pour et al. and with Springer et al. who refer to the potential for serious complications associated with hinged implants, but who do not believe that rotating knee implants are used too frequently $[12,29]$. Careful patient selection and meticulous implantation technique are preconditions for the postoperative satisfaction of patients and for a good postoperative result. To verify the clinical outcome, prospective long-term studies comparing modern-generation rotating hinge designs with implants of varying degrees of constraint are warranted.

This current study reports clinical and radiological medium to long-term results in a fairly large number of patients with RT-PLUS SOLUTION rotating hinge knee implants. The results indicate a good postoperative result. The revision rate was favourable, given the difficult patient population involved. Good clinical results were also recorded. This was reflected in the good range of motion, high level of patient satisfaction and low pain scores.

\section{References}

1. Kurtz S, Ong K, Lau E, Mowat F, Halpern M (2007) Projections of primary and revision hip and knee arthroplasty in the United States from 2005 to 2030 . J Bone Joint Surg Am 89: 780-785.

2. Lettin AW, Deliss LJ, Blackburne JS, Scales JT (1978) The stanmore hinged knee arthroplasty. J Bone Joint Surg Br 60-B: 327-332.

3. Deburge A, Guepar (1976) Guepar hinge prosthesis: complications and results with two years' follow-up. Clin Orthop Relat Res: 47-53.

4. Walker PS, Emerson R, Poter T, Scott R, Thomas WH, et al. (1982) The kinematic rotating hinge: biomechanics and clinical application. Orthop Clin North Am 13: 187-199.

5. Barrack RL, Lyons TR, Ingraham RQ, Johnson JC (2000) The use of modular rotating hinge component in salvage revision total knee arthroplasty. J Arthroplasty 15: 858-866.

6. Neumann DR, Hofstaedter T, Dorn U (2012) Follow-up of a modular rotating hinge knee system in salvage revision total knee arthroplasty. J Arthroplasty 27: 814-819.

7. Gehrke T, Kendoff D, Haasper C (2014) The role of hinges in primary total knee replacement. Bone Joint J 96-B(11 Supple A): 93-95.

8. Gudnason A, Milbrink J, Hailer NP (2011) Implant survival and outcome afte rotating-hinge total knee revision arthroplasty: a minimum 6-year follow-up. Arch Orthop Trauma Surg 131: 1601-1607.
9. Joshi N, Navarro-Quilis A (2008) Is there a place for rotating-hinge arthroplasty in knee revision surgery for aseptic loosening? J Arthroplasty 23: 1204-1211.

10. Hossain F, Patel S, Haddad FS (2010) Midterm assessment of causes and results of revision total knee arthroplasty. Clin Orthop Relat Res 468: 12211228.

11. Hwang SC, Kong JY, Nam DC, Kim DH, Park HB, et al. (2010) Revision total knee arthroplasty with a cemented posterior stabilized, condylar constrained or fully constrained prosthesis: a minimum 2-year follow-up analysis. Clin Orthop Surg 2: 112-120.

12. Pour AE, Parvizi J, Slenker N, Purtill JJ, Sharkey PF (2007) Rotating hinged total knee replacement: use with caution. J Bone Joint Surg Am 89: 17351741.

13. Lombardi AV Jr, Berend KR, Leith JR, Mangino GP, Adams JB (2007) Posterior-stabilized constrained total knee arthroplasty for complex primary cases. J Bone Joint Surg Am 89 Suppl 3: 90-102.

14. Insall JN, Dorr LD, Scott RD, Scott WN (1989) Rationale of the Knee Society clinical rating system. Clin Orthop Relat Res: 13-14.

15. Ranawat CS, Shine JJ (1973) Duo-condylar total knee arthroplasty. Clin Orthop Relat Res: 185-195.

16. Kaplan EL, Meier P (1958) Nonparametric estimation from incomplete observations. J Am Stat Assoc 53: 457-481.

17. Lawless JF (2002) Statistical models and methods for lifetime data, $2^{\text {nd }}$ edition. John Wiley \& Sons, New York.

18. Bistolfi A, Lustig S, Rosso F, Dalmasso P, Crova M, et al. (2013) Results with 98 Endo-Modell rotating hinge prostheses for primary knee arthroplasty. Orthopedics 36: e746-e752.

19. Bistolfi A, Rosso F, Crova M, Massazza G (2013) Endo-Modell rotating-hinge total knee for revision total knee arthroplasty. Orthopedics 36: e1299-e1306.

20. Bistolfi A, Massazza G, Rosso F, Crova M (2012) Rotating-hinge total knee for revision total knee arthroplasty. Orthopedics 35: e325-330.

21. Kowalczewski J, Marczak D, Synder M, Sibinski M (2014) Primary rotatinghinge total knee arthroplasty: good outcomes at mid-term follow-up. J Arthroplasty 29: 1202-1206.

22. Muller C, Basad E, Melzer C (2008) Mid term results with a new rotating hinge arthroplasty. In: American Academy of Orthopedic Surgeons (AAOS), San Francisco, CA

23. Giurea A, Neuhaus HJ, Miehlke R, Schuh R, Lass R, et al. (2014) Early results of a new rotating hinge knee implant. Biomed Res Int 2014: 948520.

24. Lozano LM, Lopez V, Rios J, Popescu D, Torner P, et al. (2012) Better outcomes in severe and morbid obese patients $\left(B M I>35 \mathrm{~kg} / \mathrm{m}^{2}\right)$ in primary Endo-Model rotating-hinge total knee arthroplasty. Scientific WorldJournal 2012: 249391.

25. Sanguineti F, Mangano T, Formica M, Franchin F (2014) Total knee arthroplasty with rotating-hinge Endo-Model prosthesis: clinical results in complex primary and revision surgery. Arch Orthop Trauma Surg 134: 16011607.

26. Barrack RL (2002) Rise of the rotating hinge in revision total knee arthroplasty. Orthopedics 25: 1020-1058

27. Rand JA, Chao EY, Stauffer RN (1987) Kinematic rotating-hinge total knee arthroplasty. J Bone Joint Surg Am 69: 489-497.

28. Callaghan JJ, O'Rourke MR, Liu SS (2005) The role of implant constraint in revision total knee arthroplasty: not too little, not too much. J Arthroplasty 20(4 Supple 2): 41-43.

29. Springer BD, Hanssen AD, Sim FH, Lewallen DG (2001) The kinematic rotating hinge prosthesis for complex knee arthroplasty. Clin Orthop Relat Res: 283-291. 